\title{
Parallel input makes the brain run faster
}

\author{
Tommi Raij,, ${ }^{\text {a,* Jari Karhu, }}$, Dubravko Kičić, c,d,e Pantelis Lioumis, ${ }^{\text {c,e }}$ Petro Julkunen,

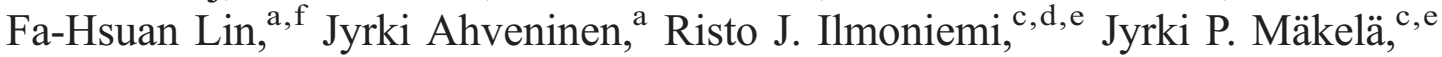 \\ Matti Hämäläinen, ${ }^{\mathrm{a}}$ Bruce R. Rosen, ${ }^{\mathrm{a}}$ and John W. Belliveau ${ }^{\mathrm{a}}$ \\ ${ }^{a}$ MGH/MIT/HMS Athinoula A. Martinos Center for Biomedical Imaging, Bldg 149, 13th St, Charlestown, MA 02129, USA \\ ${ }^{\mathrm{b}}$ Department of Clinical Neurophysiology, Kuopio University Hospital, Finland \\ ${ }^{\mathrm{c}}$ BioMag Laboratory, HUSLAB - Helsinki University Central Hospital, Finland \\ ${ }^{\mathrm{d}}$ Department of Biomedical Engineering and Computational Science, Helsinki University of Technology, Finland \\ ${ }^{\mathrm{e}}$ Helsinki Brain Research Center, Finland \\ ${ }_{\mathrm{f}}^{\mathrm{f}}$ Institute of Biomedical Engineering, National Taiwan University, Taiwan
}

Received 8 October 2007; revised 14 January 2008; accepted 18 January 2008

Available online 14 February 2008

In serial sensory processing, information flows from the thalamus via primary sensory cortices to higher-order association areas. However, association cortices also receive, albeit weak, direct thalamocortical sensory inputs of unknown function. For example, while information proceeds from primary (SI) to secondary (SII) somatosensory cortex in a serial fashion, both areas are known to receive direct thalamocortical sensory input. The present study examines the potential roles of such parallel input arrangements. The subjects were presented with median nerve somatosensory stimuli with the instruction to respond with the contralateral hand. The locations and time courses of the activated brain areas were first identified with magnetoencephalography (MEG). In a subsequent session, these brain areas were modulated with single-pulse transcranial magnetic stimulation (TMS) at 15-210 ms after the somatosensory stimulus while electroencephalography (EEG) was recorded. TMS pulses at $\mathbf{1 5}-\mathbf{4 0} \mathbf{~ m s}$ post-stimulus significantly speeded up reaction times and somatosensory-evoked responses, with largest facilitatory effects when the TMS pulse was given to contralateral SII at about $20 \mathrm{~ms}$. To explain the results, we propose that the early somatosensory-evoked physiological SII activation exerts an $\mathrm{SII} \rightarrow \mathrm{SI}$ influence that facilitates the reciprocal SI $\rightarrow$ SII pathway - with TMS to SII we apparently amplified this mechanism. The results suggest that the human brain may utilize parallel inputs to facilitate long-distance cortico-cortical connections, resulting in accelerated processing and speeded reaction times. This arrangement could also allow very early top-down modulation of the bottom-up stream of sensory information. (C) 2008 Elsevier Inc. All rights reserved.

Keywords: Brain; Human; Somatosensory; Parallel processing; Bottom-up; Top-down

\footnotetext{
* Corresponding author. Fax: +1 6177267422.

E-mail address: raij@nmr.mgh.harvard.edu (T. Raij).

Available online on ScienceDirect (www.sciencedirect.com).
}

\section{Introduction}

Serial bottom-up flow of information from sensory thalamic nuclei via primary sensory cortices to higher-order association areas has been well-established (Pons et al., 1987). However, direct thalamocortical inputs bypassing the primary sensory cortices also exist. In non-human primates, direct input to the secondary somatosensory cortex SII (Kaas and Garraghty, 1991; Zhang et al., 2001, 1996) and crossmodal inputs to islets in sensory association cortices (Schroeder et al., 2001) have been reported. In humans, higher-order cortices may become activated even earlier than primary sensory cortices (Barba et al., 2002; ffytche et al., 1995; Karhu and Tesche, 1999), which suggests parallel pathway arrangements. However, the functional roles of parallel sensory inputs to association cortices are unknown. The current study examines the possible advantages of such inputs. Specifically, inspired by the "counter streams" theory of visual processing (Ullman, 1995, 1996), we hypothesized that they facilitate corticocortical communications between primary sensory cortex and the higher-order cortical areas that receive parallel inputs directly from the thalamus.

To this aim, we first presented somatosensory median nerve stimuli with a reaction time (RT) task while measuring the brain activations with magnetoencephalography (MEG). This provided the locations and timings of the activated somatomotor network. In a subsequent session, the identified brain areas were then modulated with a transcranial magnetic stimulation (TMS) pulse at different latencies after the somatosensory stimulus. The resulting modulations were detected with simultaneous RT and electroencephalographic (EEG) recordings. We hypothesized that a TMS pulse given immediately after the somatosensory stimulus would speed up brain processing and RTs. Moreover, we anticipated that the RT advantage would be greatest when higher-order cortical 
areas, rather than the primary somatosensory cortex, were stimulated with TMS.

\section{Materials and methods}

\section{Subjects, stimuli, and task}

The subjects were three healthy human males (age 26-41 years, one left-handed). The somatosensory stimuli were 0.2 -ms electrical impulses to the dominant hand median nerve, generating a visible thumb twitch. To preclude anticipatory effects, the interstimulus interval was variable (mean $2.3 \mathrm{~s}$, range 1.5-21 s). The experiment was conducted in 4-min runs, each containing 40 stimuli/responses. The task was to respond to each stimulus with the index finger of the non-dominant hand (contralateral to the somatosensory stimulus) as quickly as possible while RT was measured. Outlier RTs $(4.3 \%)$ were removed based on falling outside mean \pm 2 SD across all runs.

\section{Magnetic resonance imaging (MRI)}

Structural T1-weighted images were obtained with a 1.5-T Siemens Allegra (Siemens, Germany) scanner and segmented with the FreeSurfer (Fischl et al., 2002) software (http://surfer.nmr.mgh. harvard.edu).

\section{Experiment 1: $M E G$}

Whole-head 306-channel MEG was recorded with a VectorView neuromagnetometer (Elekta Neuromag, Finland) at $0.01-330 \mathrm{~Hz}$ and sampled at $1 \mathrm{kHz}$. Responses from 120 trials were averaged with respect to the somatosensory stimuli to reveal event-related fields (ERFs); epochs containing electro-oculogram (EOG) signals exceeding $\pm 150 \mu \mathrm{V}$ were discarded. The generators of the ERFs were located using dipole modeling. The dipole amplitudes were then allowed to vary in a multidipole model as a function of time while keeping their locations and orientations fixed. This resulted in

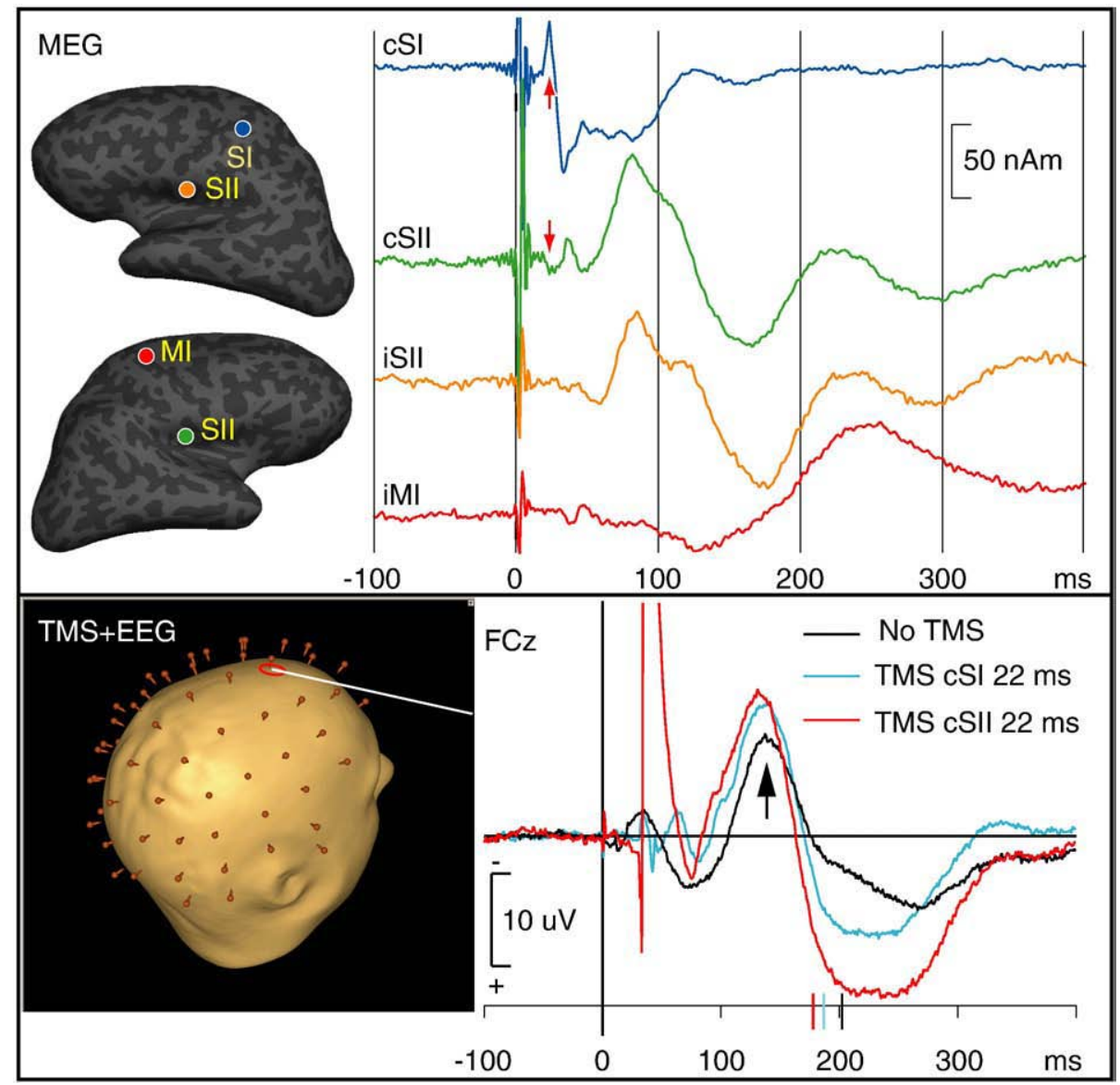

Fig. 1. Upper panel: MEG experiment. MEG source locations, shown on inflated cortex, and time courses from a typical subject. The subject responded to right median nerve stimuli with the left index finger. The evoked MEG responses were generated by four sources: the primary somatosensory cortex in the hemisphere contralateral to the median nerve stimulus (cSI), the secondary somatosensory cortices bilaterally (cSII, iSII), and the primary motor cortex contralateral to the motor response (but ipsilateral to the median never stimulus, iMI). In the time courses, a somatosensory stimulator artifact is observed at time 0 . The cSI waveform showed maxima at $23 \mathrm{~ms}$ (upper red arrow) $/ 33 \mathrm{~ms}$, while cSII showed early activity already at about 20-35 ms (lower red arrow) and major peaks at 90/170 ms. The iSII exhibited similar 90/170 ms deflections as cSII. The iMI showed typical motor-evoked activity with a maximum slightly after the movement onset at $210 \mathrm{~ms}$. Lower panel: TMS +EEG experiment. Somatosensory ERPs (unfiltered grand average waveforms) to identical stimuli and task as above. Responses recorded from the midline frontal location FCz were selected for display; according to our simulations these mainly reflect bilateral SII activity. Compared to the condition without TMS (black line), the two shown TMS conditions (blue and red lines) reveal earlier and stronger SII activity already at $\sim 140$ ms (black arrow). The ERP time shifts appear to correspond to the speeded RTs (ticks on the ERP time scales). 
millisecond-accuracy time courses of the activated brain areas (Hämäläinen and Hari, 2002).

\section{Experiment 2: Navigated TMS and EEG}

Single-pulse TMS (Ilmoniemi et al., 1999) was delivered with a Magstim Rapid stimulator (Magstim Company, UK) and figure-ofeight coil (Magstim 9925) navigated with eXimia NBSTM (Nexstim Ltd., Finland) to target the brain areas identified with MEG. TMS intensity was $120 \%$ of the subject-specific motor threshold. A total of 25-31 runs were recorded per subject (including three without TMS pulses) resulting in 1040-1240 trials per subject. To probe different stages of processing, the TMS pulse latency across runs was varied $15-210 \mathrm{~ms}$ after the somatosensory stimulus, with the TMS latencies tailored for each subject individually based on their MEG responses. The order of TMS latencies in each brain location was randomized. Simultaneous EEG was recorded using a 60channel TMS-compatible eXimia EEG system (Nexstim), bandpass filtered at $0.1-350 \mathrm{~Hz}$, and sampled at $1.45 \mathrm{kHz}$ at 16 -bit depth (mean reference). The EEG amplifiers were decoupled from the electrodes for $9 \mathrm{~ms}$ during delivery of the TMS pulse. The EEG responses were averaged with respect to the somatosensory stimuli to reveal event-related potentials (ERPs) separately for each TMS location and latency. Epochs contaminated by eye blinks were discarded using $\pm 100 \mu \mathrm{V}$ threshold. EEG sensor locations that best reflected the activity of cSI, cSII, iSII, and iMI were determined by forward modeling the MEG data to simulate corresponding ERPs. Peak ERP latencies were then identified for somatosensory-evoked N20/P45/P75/N140 components separately for each TMS location and latency (provided that the strong TMS-evoked response did not distort the somatosensory-evoked component beyond recognition).

\section{Results}

First, MEG (Fig. 1, upper panel) revealed expected (Hari and Forss, 1999) sources and their activation time courses in the contralateral primary somatosensory cortex (cSI), SIIs bilaterally (cSII, iSII), and ipsilateral motor cortex (iMI). Simulations suggested that the observed cSII activity at about $20-35 \mathrm{~ms}$ could not be explained by volume conduction from unaccounted cSI sources.

Second, in a subsequent session, processing in these four brain areas was modulated with a single TMS pulse at 15-210 ms after the somatosensory stimulus while EEG and RT were recorded (Fig. 1, lower panel). Without TMS pulses, RT was $203 \pm 29 \mathrm{~ms}$ (mean $\pm \mathrm{SD}$, collapsed across subjects; means of individual subjects had a range of 197-209 ms).

Fig. 2 shows that the TMS pulse after the somatosensory stimulus clearly effected RTs. In each of the four targeted brain areas, TMS pulse latency was positively correlated with RT. The linear correlation was strongest in cSII (Pearson's correlation $r=0.83$ ), somewhat weaker in iSII $(r=0.74)$ and $\mathrm{cSI}(r=0.74)$, and weakest in iMI cortex $(r=0.54)$. Lack of correlation between run order and RT suggested that fatigue did not play a role (for individual subjects, Pearson's $r$ ranged from -0.17 to +0.13 ).

Prolonged RTs demonstrated that the TMS pulse interfered with the neuronal processes. As hypothesized, early TMS pulses (15$40 \mathrm{~ms}$ after the somatosensory stimulus) were associated with significantly faster RTs than without TMS (Student's 2-tailed heterosce-

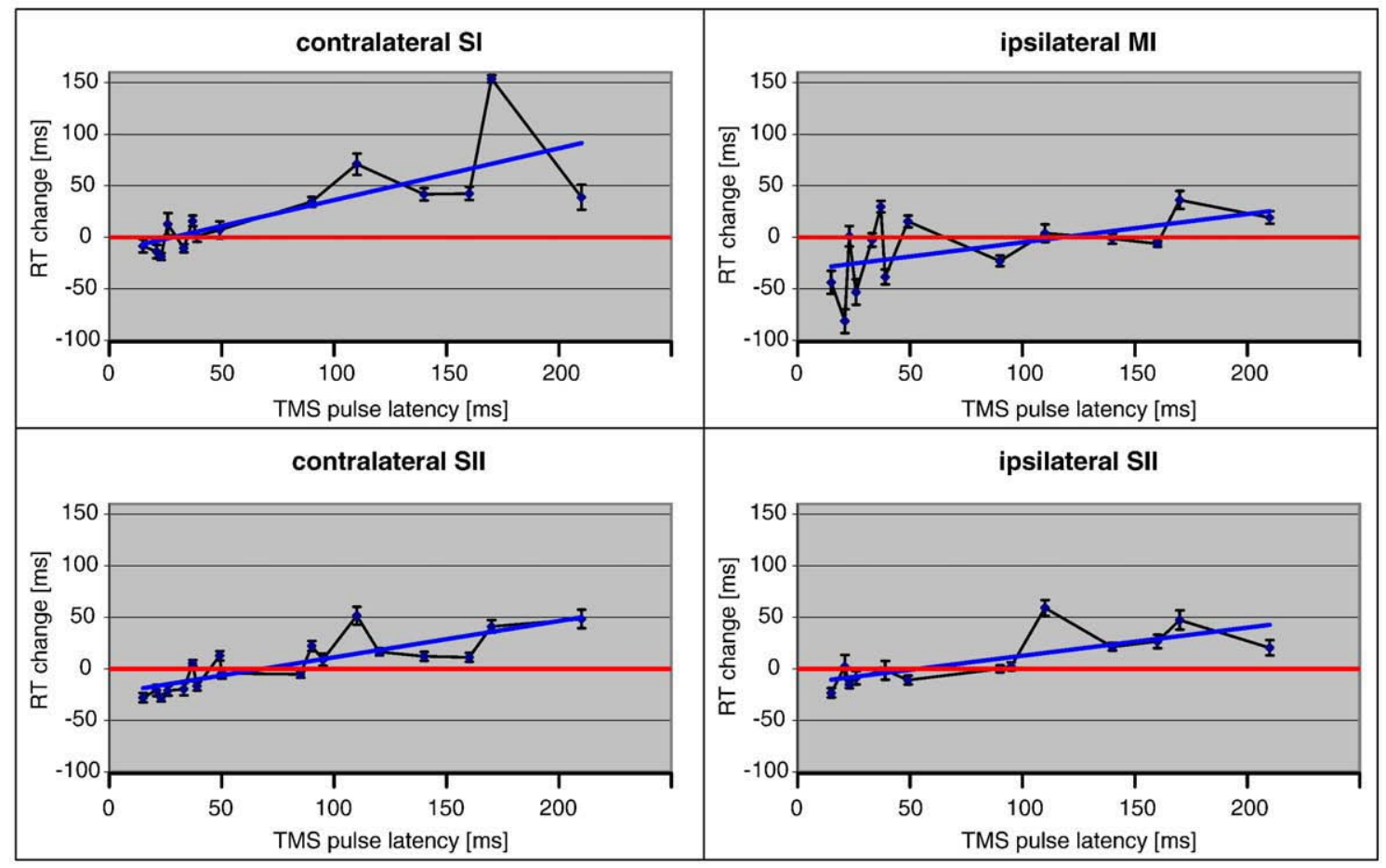

Fig. 2. Reaction time change $(0 \mathrm{~ms}=\mathrm{RT}$ without TMS $)$ as a function of TMS pulse latency $(0 \mathrm{~ms}=$ Median nerve somatosensory stimulus $)$. The panels show the effects separately for the four brain areas that were targeted with TMS. All brain areas showed prolonged RTs with increasing TMS pulse latency (blue trend lines), with the strongest linear correlation with TMS of the contralateral SII (lower left panel). The earliest TMS pulse latencies were associated with significantly faster RTs than without TMS. While this effect was observed in each of the four brain areas, it was clearest for ipsilateral MI (contralateral with respect to the reaction key hand) and for contralateral SII. Data collapsed across subjects, mean \pm SEM error bars. For details, see text. 
dastic $t$-test $p<0.001$; collapsed across all four brain areas from all subjects at TMS latencies 15-40 ms; for each TMS target area individually $p<0.05$ ). The motor cortex (iMI; Fig. 2, upper right panel) showed the largest RT speeding effects, but this may have occurred simply because TMS activated the motor system regardless of the somatosensory stimulus. However, the earliest (15-23 ms) TMS pulses to $\mathrm{cSI}(\mathrm{RT}=189 \pm 35 \mathrm{~ms}), \mathrm{cSII}(\mathrm{RT}=176 \pm 27 \mathrm{~ms})$, or iSII $(\mathrm{RT}=187 \pm 42 \mathrm{~ms})$ also significantly speeded up the RTs $(p<0.001$ for each area separately collapsed across subjects; for cSII $p<0.001$ for each subject individually). This effect was TMS location-specific: supporting our hypothesis, RT was significantly faster when TMS was given to cSII than to cSI ( $p<0.001$; collapsed across subjects at TMS latencies $15-23 \mathrm{~ms})$ or to iSII $(p<0.01)$.

We then analyzed the ERP data to understand at which level of processing the RT speeding effect occurred. Fig. 1, lower panel, shows that the latency of the SII-generated $\sim 140 \mathrm{~ms}$ component was shifted earlier with a TMS pulse at $\sim 20 \mathrm{~ms}$ after the somatosensory stimulus. Peak latency analyses revealed that TMS pulses at 15$40 \mathrm{~ms}$ speeded the $140-\mathrm{ms}$ ERP component by $8 \pm 8 \mathrm{~ms}$ compared with the no-TMS condition. This effect was statistically significant ( $p<0.05$; collapsed across brain areas and subjects). The ERP data were thus consistent with the idea that the brain activations were speeded already at the SII level. The ERP waveforms selected for display in Fig. 1, lower panel, further suggests that, similar to the RT data, the largest latency shifts were observed when TMS was targeted at cSII; however, in the ERP data, this trend did not reach statistical significance.

Compared with the time window when TMS pulses speeded RTs (15-40 ms), the observed ERP latency shift at $\sim 140$ ms appears a relatively late phenomenon. However, the strong TMS-evoked ERPs, maximal under the TMS target location and lasting $\sim 100 \mathrm{~ms}$ after the TMS pulse, resulted in that, of the somatosensory-evoked components, only the 140-ms deflection could be reliably identified across experimental conditions. It is thus possible that the somatosensory system latency shifts started before $140 \mathrm{~ms}$ but our paradigm could not detect them. Future studies may benefit from a subtraction technique that allows separation of the sensory- and TMS-evoked components (Thut et al., 2003, 2005).

\section{Discussion}

We observed speeded RTs and somatosensory-evoked responses when a TMS pulse was delivered to the somatomotor network 15$40 \mathrm{~ms}$ after a median nerve stimulus. Largest facilitatory effects were observed when the TMS pulse was targeted at the contralateral SII at about $20 \mathrm{~ms}$ post-stimulus.

Previous studies utilizing human intracranial recordings have shown SII activity beginning already at $15-27 \mathrm{~ms}$ post-stimulus, which is simultaneous or earlier than onset of the SI activity (Barba et al., 2002). Therefore, SII must receive direct early parallel sensory input independent of the pathway via SI, consistent with the current and earlier (Karhu and Tesche, 1999) MEG observations.

Speeded RTs for TMS at early post-stimulus latencies have been described before (Gregori et al., 2005), thus supporting our behavioral results. However, these effects have been attributed to multisensory redundancy caused by the auditory click from the stimulator coil, largely because in these studies the RT effect has been similar regardless of the TMS target area (Gregori et al., 2005; Walsh and Pascual-Leone, 2003). Our results show TMS site specificity and therefore are not compatible with this interpretation.
To examine this further, we made control measurements in one subject where the auditory click from the TMS coil was identical but the TMS-evoked currents were reduced by over $50 \%$ (sham coil). The TMS pulse was given $21 \mathrm{~ms}$ after the somatosensory stimulus. The subject did not know when real vs. sham TMS was used. RTs were significantly faster for real vs. sham stimulation over cSII $(p<0.05)$ and iMI $(p<0.001)$ but not over cSI $(p=0.15)$; iSII was not tested. Both TMS site specificity and the control measurement therefore support the idea that the speeded RTs were caused by TMS-evoked neuronal currents.

We propose that the speeded RTs can be best explained if the somatosensory-evoked physiological SII activation at about $20 \mathrm{~ms}$ normally exerts a top-down $\mathrm{SII} \rightarrow$ SI influence that facilitates the reciprocal SI $\rightarrow$ SII pathway. With TMS to SII at $\sim 20 \mathrm{~ms}$, it appears that we amplified a brain-speeding mechanism already in place. This interpretation is supported with the current findings of site specificity of TMS and ERP latency shifts already at the SII level.

More generally, fast thalamocortical parallel sensory inputs to multiple cortical sites could drop the activation thresholds of the cortico-cortical connections between the areas (Ullman, 1996). This mechanism could almost immediately after a stimulus establish a widespread network where the nodes receiving parallel input would be likely to communicate with each other.

Theoretical and physiological studies have suggested that topdown effects may facilitate and guide the reciprocal bottom-up flow, even though the cellular-level mechanisms are still poorly known (Siegel et al., 2000; Ullman, 1995, 1996). However, in order to be effective, top-down processes should be running already when the bottom-up stream is finding its way towards higher levels of cortical hierarchy (Ullman, 1996). This is obviously difficult to achieve with serial processing. One possibility is that the brain utilizes serial pathways specialized for very fast information transfer to initiate early activity in high-order association cortices. For example, visual recognition has been shown to utilize early top-down influences from orbitofrontal cortex initiated by fast serial (via V1) magnocellular pathways (Bar, 2003; Bar et al., 2006; Kveraga et al., 2007). The somatosensory data are inconsistent with serial processing models because activations start earlier in SII than SI. The current study therefore offers an appealing alternative mechanism: association cortices could receive direct thalamocortical sensory input, allowing simultaneous top-down and bottom-up processing. Both mechanisms may well coexist.

The idea of parallel thalamocortical sensory inputs to multiple cortical areas may appear inconsistent with the view that transmission of sensory information is hard wired from the thalamic sensory nucleus to the corresponding sensory projection cortex. However, first-order thalamic nuclei receiving driving input from sensory organs are reciprocally connected with and heavily modulated by both higher-order thalamic nuclei (e.g., pulvinar) and cortex, reflecting attentional and other task-related demands (Guillery and Sherman, 2002; O'Connor et al., 2002; Sherman, 2007; Sherman and Guillery, 1996, 2002; see Bender and Youakim, 2001; Briggs and Usrey, 2007; Zikopoulos and Barbas, 2007 for recent related work in primates). Pulvinar, on the other hand, has massive reciprocal connections throughout the neocortex (e.g., Adams et al., 2000; Buchsbaum et al., 2006; see Shipp, 2003 for a review). The cortex can thus receive fast sensory input from the thalamus directly from the first-order thalamic nucleus (when such pathways exist) or through a higher-order thalamic area such as the pulvinar. Modulating inputs to thalamic nuclei could in a dynamic manner adjust which cortical areas receive parallel sensory input. 
Given that both association and low-level sensory cortices appear to receive very early parallel crossmodal inputs (Fort et al., 2002, 2000; Foxe and Schroeder, 2005; Giard and Peronnet, 1999; Molholm et al., 2002; Murray et al., 2005; Schroeder and Foxe, 2002, 2005; Schroeder et al., 2003), some via the pulvinar (Budinger et al., 2006; Hackett et al., 2007), a similar mechanism as suggested in the current study could also explain why reaction times to multisensory stimuli are faster than to unisensory stimuli (Raab, 1962; Schröger and Widmann, 1998). Early physiological SII activations may also serve a protective function due to the roles of SII in pain processing (Timmermann et al., 2001) and sensorimotor integration (Forss and Jousmäki, 1998; Huttunen et al., 1996).

It has been suggested that serial processing is more prevalent in higher primates, and there seems to be an evolutionary shift in mammals where humans have the least amount of parallel sensory inputs to higher-order areas (Coleman et al., 1999; Kaas and Garraghty, 1991; Zhang et al., 2001, 1996) and therefore increased serial processing of sensory input. Thus, it appears that in the course of evolution humans may have traded some processing speed for better cognitive control.

From the large number of trials and consistent results across subjects, it follows that the current results are reliable within the studied population, but due to limited access (the instruments were located on different continents), our number of subjects was small. Hence, more studies with larger subject populations are needed to estimate how abundant this mechanism is.

\section{Concluding remarks}

The cerebral cortex receives sensory input from the thalamus not only to primary projection areas but also directly to hierarchically higher-order cortices in a parallel fashion. The current results suggest that this facilitates cortico-cortical communication between the areas that receive parallel input, thus making the brain faster. This also allows very early top-down modulation of the bottom-up stream of sensory input. The same mechanism could drop the activation thresholds between the participating cortical nodes, therefore establishing a distributed neuronal network almost immediately after a stimulus. Further studies are needed.

\section{Acknowledgments}

The authors thank Moshe Bar, Rozalya Bikmullina, Deirdre Foxe, John Foxe, Riitta Hari, Hsiao-Wen Huang, Yu-Hua Huang, Ted Huppert, Iiro Jääskeläinen, G.W. Krauss, Alvaro PasqualLeone, Cherif Sahyoun, Dahlia Sharon, and Linda Stenbacka for help and comments. This work was supported by US National Institutes of Health Grants R01 NS048279, R01 HD040712, R01 NS037462, P41 RR14075, R21 EB007298, National Center for Research Resources, Sigrid Juselius Foundation, Academy of Finland, Finnish Cultural Foundation, Instrumentarium Science Foundation, Taiwan National Science Council NSC 96-2320-B002-085, and Taiwan National Health Research Institute 29C97N.

\section{References}

Adams, M.M., Hof, P.R., Gattass, R., Webster, M.J., Ungerleider, L.G., 2000. Visual cortical projections and chemoarchitecture of macaque monkey pulvinar. J. Comp. Neurol. 419, 377-393.

Bar, M., 2003. A cortical mechanism for triggering top-down facilitation in visual object recognition. J. Cogn. Neurosci. 15, 600-609.
Bar, M., Kassam, K.S., Ghuman, A.S., Boshyan, J., Schmid, A.M., Dale, A.M., Hämäläinen, M.S., Marinkovic, K., Schacter, D.L., Rosen, B.R., Halgren, E., 2006. Top-down facilitation of visual recognition. Proc. Natl. Acad. Sci. U.S.A. 103, 449-454.

Barba, C., Frot, M., Mauguiere, F., 2002. Early secondary somatosensory area (SII) SEPs. Data from intracerebral recordings in humans. Clin. Neurophysiol. 113, 1778-1786.

Bender, D.B., Youakim, M., 2001. Effect of attentive fixation in macaque thalamus and cortex. J. Neurophysiol. 85, 219-234.

Briggs, F., Usrey, W.M., 2007. A fast, reciprocal pathway between the lateral geniculate nucleus and visual cortex in the macaque monkey. J. Neurosci. 27, 5431-5436.

Buchsbaum, M.S., Buchsbaum, B.R., Chokron, S., Tang, C., Wei, T.C., Byne, W., 2006. Thalamocortical circuits: fMRI assessment of the pulvinar and medial dorsal nucleus in normal volunteers. Neurosci. Lett. 404, 282-287.

Budinger, E., Heil, P., Hess, A., Scheich, H., 2006. Multisensory processing via early cortical stages: connections of the primary auditory cortical field with other sensory systems. Neuroscience 143, 1065-1083.

Coleman, G.T., Zhang, H.Q., Murray, G.M., Zachariah, M.K., Rowe, M.J., 1999. Organization of somatosensory areas I and II in marsupial cerebral cortex: parallel processing in the possum sensory cortex. J. Neurophysiol. 81, 2316-2324.

ffytche, D.H., Guy, C.N., Zeki, S., 1995. The parallel visual motion inputs into areas V1 and V5 of human cerebral cortex. Brain 118, 1375-1394.

Fischl, B., Salat, D.H., Busa, E., Albert, M., Dieterich, M., Haselgrove, C., van der Kouwe, A., Killiany, R., Kennedy, D., Klaveness, S., Montillo, A., Makris, N., Rosen, B., Dale, A.M., 2002. Whole brain segmentation: automated labeling of neuroanatomical structures in the human brain. Neuron. 33, 341-355.

Forss, N., Jousmäki, V., 1998. Sensorimotor integration in human primary and secondary somatosensory cortices. Brain Res. 781, 259-267.

Fort, A., Delpuech, C., Pernier, J., Giard, M-H., 2002. Early auditory-visual interactions in human cortex during nonredundant target identification. Brain Res. Cogn. Brain Res. 14, 20-30.

Foxe, J.J., Schroeder, C.E., 2005. The case for feedforward multisensory convergence during early cortical processing. NeuroReport 16, 419-423.

Foxe, J.J., Morocz, I.A., Murray, M.M., Higgins, B.A., Javitt, D.C., Schroeder, C.E., 2000. Multisensory auditory-somatosensory interactions in early cortical processing revealed by high-density electrical mapping. Brain Res. Cogn. Brain Res. 10, 77-83.

Giard, M.H., Peronnet, F., 1999. Auditory-visual integration during multimodal object recognition in humans: a behavioral and electrophysiological study. J. Cogn. Neurosci. 11, 473-490.

Gregori, B., Curra, A., Dinapoli, L., Bologna, M., Accornero, N., Berardelli, A., 2005. The timing and intensity of transcranial magnetic stimulation, and the scalp site stimulated, as variables influencing motor sequence performance in healthy subjects. Exp. Brain Res. 166, 43-55.

Guillery, R.W., Sherman, S.M., 2002. Thalamic relay functions and their role in corticocortical communication: generalizations from the visual system. Neuron. 33, 163-175.

Hackett, T.A., De La Mothe, L.A., Ulbert, I., Karmos, G., Smiley, J., Schroeder, C.E., 2007. Multisensory convergence in auditory cortex: II. Thalamocortical connections of the caudal superior temporal plane. J. Comp. Neurol. 502, 924-952.

Hämäläinen, M.S., Hari, R., 2002. Magnetoencephalographic characterization of dynamic brain activation. Basic principles and methods of data collection and source analysis. In: Toga, A.W. (Ed.), Brain Mapping: The Methods. Academic Press, New York, pp. 227-253.

Hari, R., Forss, N., 1999. Magnetoencephalography in the study of human somatosensory cortical processing. Philos. Trans. R. Soc. Lond., B. Biol. Sci. 354, 1145-1154.

Huttunen, J., Wikström, H., Korvenoja, A., Seppäläinen, A.M., Aronen, H., Ilmoniemi, R.J., 1996. Significance of the second somatosensory cortex in sensorimotor integration: enhancement of sensory responses during finger movements. NeuroReport 7, 1009-1012. 
Ilmoniemi, R.J., Ruohonen, J., Karhu, J., 1999. Transcranial magnetic stimulation - a new tool for functional imaging of the brain. Crit. Rev. Biomed. Eng. 27, 241-284.

Kaas, J.H., Garraghty, P.E., 1991. Hierarchical, parallel, and serial arrangements of sensory cortical areas: connection patterns and functional aspects. Curr. Opin. Neurobiol. 1, 248-251.

Karhu, J., Tesche, C.D., 1999. Simultaneous early processing of sensory input in human primary (SI) and secondary (SII) somatosensory cortices. J. Neurophys. 81, 2017-2025.

Kveraga, K., Boshyan, J., Bar, M., 2007. Magnocellular projections as the trigger of top-down facilitation in recognition. J. Neurosci. 27, 13232-13240.

Molholm, S., Ritter, W., Murray, M.M., Javitt, D.C., Schroeder, C.E., Foxe, J.J., 2002. Multisensory auditory-visual interactions during early sensory processing in humans: a high-density electrical mapping study. Brain Res. Cogn. Brain. Res. 14, 115-128.

Murray, M.M., Molholm, S., Michel, C.M., Heslenfeld, D.J., Ritter, W., Javitt, D.C., Schroeder, C.E., Foxe, J.J., 2005. Grabbing your ear: rapid auditory-somatosensory multisensory interactions in low-level sensory cortices are not constrained by stimulus alignment. Cereb. Cortex 15 , 963-974.

O’Connor, D.H., Fukui, M.M., Pinsk, M.A., Kastner, S., 2002. Attention modulates responses in the human lateral geniculate nucleus. Nat. Neurosci. 5, 1203-1209.

Pons, T.P., Garraghty, P.E., Friedman, D.P., Mishkin, M., 1987. Physiological evidence for serial processing in somatosensory cortex. Science $237,417-420$

Raab, D.H., 1962. Statistical facilitation of simple reaction times. Trans. N.Y. Acad. Sci. 24, 574-590.

Schroeder, C.E., Foxe, J.J., 2002. The timing and laminar profile of converging inputs to multisensory areas of the macaque neocortex. Brain Res. Cogn. Brain Res. 14, 187-198.

Schroeder, C.E., Foxe, J.J., 2005. Multisensory contributions to low-level, 'unisensory' processing. Curr. Opin. Neurobiol. 15, 454-458.

Schroeder, C.E., Lindsley, R.W., Specht, C., Marcovici, A., Smiley, J.F., Javitt, D.C., 2001. Somatosensory input to auditory association cortex in the macaque monkey. J. Neurophysiol. 85, 1322-1327.

Schroeder, C.E., Smiley, J., Fu, K.G., McGinnis, T., O, O’Connell, M.N., Hackett, T.A., 2003. Anatomical mechanisms and functional implications of multisensory convergence in early cortical processing. Int. J. Psychophysiol. 50, 5-17.

Schröger, E., Widmann, A., 1998. Speeded responses to audiovisual signal changes result from bimodal integration. Psychophysiology 35, 755-759.
Sherman, S.M., 2007. The thalamus is more than just a relay. Curr. Opin. Neurobiol. 17, 417-422.

Sherman, S.M., Guillery, R.W., 1996. Functional organization of thalamocortical relays. J. Neurophysiol. 76, 1367-1395.

Sherman, S.M., Guillery, R.W., 2002. The role of the thalamus in the flow of information to the cortex. Philos. Trans. R. Soc. Lond., B. Biol. Sci. 357, $1695-1708$.

Shipp, S., 2003. The functional logic of cortico-pulvinar connections. Philos. Trans. R. Soc. Lond., B. Biol. Sci. 358, 1605-1624.

Siegel, M., Körding, K.P., König, P., 2000. Integrating top-down and bottomup sensory processing by somato-dendritic interactions. J. Comput. Neurosci. 8, 161-173.

Thut, G., Northoff, G., Ives, J.R., Kamitani, Y., Pfennig, A., Kampmann, F., Schomer, D.L., Pascual-Leone, A., 2003. Effects of single-pulse transcranial magnetic stimulation (TMS) on functional brain activity: a combined event-related TMS and evoked potential study. Clin. Neurophysiol. 114, 2071-2080.

Thut, G., Ives, J.R., Kampmann, F., Pastor, M.A., Pascual-Leone, A., 2005. A new device and protocol for combining TMS and online recordings of EEG and evoked potentials. J. Neurosci. Methods 141, 207-217.

Timmermann, L., Ploner, M., Haucke, K., Schmitz, F., Baltissen, R., Schnitzler, A., 2001. Differential coding of pain intensity in the human primary and secondary somatosensory cortex. J. Neurophysiol. 86, $1499-1503$.

Ullman, S., 1995. Sequence seeking and counter streams: a computational model for bidirectional information flow in the visual cortex. Cereb. Cortex 5, 1-11.

Ullman, S., 1996. Sequence seeking and counter streams: a model for visual cortex. In: Ullman, S. (Ed.), High-Level Vision: Object Recognition and Visual Cognition. MIT Press, Cambridge MA.

Walsh, V., Pascual-Leone, A., 2003. Transcranial Magnetic Stimulation A Neurochronometrics of Mind. MIT Press, Cambridge, MA.

Zhang, H.Q., Murray, G.M., Turman, A.B., Mackie, P.D., Coleman, G.T., Rowe, M.J., 1996. Parallel processing in cerebral cortex of the marmoset monkey: effect of reversible SI inactivation on tactile responses in SII. J. Neurophysiol. 76, 3633-3655.

Zhang, H.Q., Murray, G.M., Coleman, G.T., Turman, A.B., Zhang, S.P., Rowe, M.J., 2001. Functional characteristics of the parallel SI- and SIIprojecting neurons of the thalamic ventral posterior nucleus in the marmoset. J. Neurophysiol. 85, 1805-1822.

Zikopoulos, B., Barbas, H., 2007. Parallel driving and modulatory pathways link the prefrontal cortex and thalamus. PLoS ONE 2, e848. 